\title{
Tissue Expression of Erythropoietin Predicts Survival Rates in Clear Cell Renal Cell Carcinoma
}

\author{
Daniel Beltrame Ferreira ${ }^{\mathrm{a}, *}$, Walter Henriques da Costa ${ }^{\mathrm{a}}$, Diego Abreu Clavijo ${ }^{\mathrm{c}}$, Ricardo Decia $^{\mathrm{c}}$, \\ Isabela Werneck Cunha ${ }^{\mathrm{b}, \mathrm{d}}$, Luciana Schultz ${ }^{\mathrm{d}}$, Rafael Malagoli Rocha ${ }^{\mathrm{d}}$, \\ Gustavo Cardoso Guimarães ${ }^{\mathrm{e}}$ and Stênio de Cássio Zequi ${ }^{\mathrm{a}, \mathrm{b}}$ \\ ${ }^{a}$ Urology Division, Department of Pelvic Surgery, AC Camargo Cancer Center, São Paulo, Brazil \\ ${ }^{\mathrm{b}}$ National Institute for Science and Technology in Oncogenomics and Therapeutic Innovation \\ ${ }^{\mathrm{c}}$ Department of Urology, Pasteur Hospital, Montevideo, Uruguay \\ ${ }^{\mathrm{d}}$ Department of Anatomic Pathology, AC Camargo Cancer Center, São Paulo, Brazil \\ ${ }^{\mathrm{e}}$ Chairman of Urology Division, Department of Pelvic Surgery, AC Camargo Cancer Center, São Paulo, Brazil
}

\begin{abstract}
.
Objective: To evaluate immunohistochemical erythropoietin (EPO) expression in clear cell renal cell carcinoma (ccRCC), its association with major clinicopathological variables and its prognostic impact.

Methods: A total of 220 patients with renal cell carcinoma (RCC) surgically treated between 1989 and 2009 were evaluated in this multi-institutional study. All the cases were reviewed by a single pathologist and the immunohistochemical reactivity to EPO was analysed using tissue microarray.

Results: A total of 176 patients with ccRCC were considered, with an average of 48 months of follow-up. Of the tumours evaluated, 47 (26.7\%) were negative for EPO expression, and 129 (73.3\%) were positive. EPO expression was associated with incidental tumour $(p=0.016)$, tumour size $(p=0.015)$, Karnofsky Performance Score (KPS) $(p=0.016)$, blood transfusion $(p=0.009)$ and adrenal involvement $(p=0.038)$. The median ages of the patients with positive and negative EPO expression were 56.2 years and 66.6 years. Immunohistochemical EPO expression affected overall survival (OS) and disease-specific survival (DSS) rates. The DSS rates of the patients whose tissue was positive and negative for EPO expression were $85.3 \%$ and $76.1 \%$, respectively $(p=0.044)$. In a multivariate analysis, the absence of EPO expression proved to be a bad prognostic factor and negatively affected the OS $(p<0.001)$ and DSS $(p<0.001)$ rates.

Conclusion: The absence of tumour EPO expression is an independent predictive factor with a negative effect on survival rates. The use of EPO as possible marker in the management of ccRCC patients requires further studies and a better understanding of the role of EPO in tumour biology.
\end{abstract}

Keywords: Biomarkers, EPO, oncology, prognosis, renal cell carcinoma

\section{INTRODUCTION}

Renal cell carcinoma (RCC) accounts for 3\% of

${ }^{*}$ Correspondence to: Daniel Beltrame Ferreira, Professor Antônio Prudente, 211 - Liberdade - São Paulo - SP, ZIP CODE 01509 - 010, Brazil. Tel.: +55 112709 8136; E-mail: drbeltrame.uro@gmail.com. malignant adult cancers, and its incidence has been increasing over the last 20 years [1-4]. In Europe, 115,000 new cases were estimated in 2012, with 49,000 deaths occurring as a result of the disease [5]. 
RCC is a tumour with a heterogeneous presentation and unpredictable evolution, despite improvements in diagnostic methods and treatment [6].

Historically, the development of prognostic models based on clinical, anatomical and histopathological variables has been considered important in the treatment of RCC. However, more accurate prognostic factors can improve accuracy in predicting results and aid in individualising the treatment of these patients. Advancements in genomics and proteomics have enabled the identification of key molecular pathways involved in the growth and development of RCC metastases. In recent years, therefore, numerous molecular and genetic markers have provided a better understanding of tumour biology and have been studied as prognostic factors [7].

Recently, erythropoietin (EPO) and its receptor (EPO-R) have been found in several nonhaematopoietic tissues, such as the liver, uterus, central nervous system, endothelial cells and various malignant solid tumours, including RCC $[8,9]$. The effects of EPO on stimulating erythropoiesis are known; however, in tumours and non-haematopoietic tissues, it is believed that this hormone can stimulate angiogenesis, promote cell proliferation and inhibit apoptosis $[10,11]$. These findings have aroused interest in studying the effects of EPO on such tissues, primarily with respect to the use of recombinant human erythropoietin (rhEPO) in the treatment of anaemia that is present in many neoplasias. Moreover, the majority of renal tumours originate in the proximal tubules of the renal cortex, which is recognised as a site of EPO production in adults [12-14].

Although many studies have demonstrated EPO expression in RCC, the prognostic significance of the expression of this marker is still unclear and studies have yielded conflicting results [15]. Therefore, the aim of the present study was to immunohistochemically evaluate EPO expression in clear cell RCC (ccRCC) and adjacent benign tissue, the association of this expression with major clinicopathological variables and its prognostic impact.

\section{PATIENTS AND METHODS}

A total of 220 patients who underwent radical or partial nephrectomy at two South American centres were evaluated between 1989 and 2009. Both institutions are members of the LARCG (Latin American Renal Cancer Group) [16], and clinical data were obtained from their medical records. The pathological data were reviewed and standardised by a single experienced pathologist (IWC), and the most representative areas of the tumour were selected for the construction of a tissue microarray (TMA). A follow-up was performed every 3 months during the first 2 years, followed by every 6 months until the fifth year and yearly thereafter. The evaluation of metastases was performed with computerised tomography (CT) or magnetic resonance imaging (MRI) of the abdomen and the pelvis, a chest $\mathrm{X}$-ray, or a bone scan of the entire body. Patients with other malignancies, those undergoing neoadjuvant treatment, those with incomplete data and those lost during follow-up were excluded. Only ccRCC cases were considered in the present study, providing a final sample of 176 patients. The study was approved by the Brazilian National Research Ethics Commission ( $\mathrm{n}^{\circ}$ 549/2011).

The clinicopathological variables included were age, gender, smoking status, American Society of Anesthesiologists (ASA) classification, symptoms present at diagnosis, surgery type, staging (TNM AJCCC/UICC 2010), Fuhrman grade, histological subtype (WHO Classification/2004), vascular and lymphatic invasion, presence of necrosis, perirenal fat invasion, lymph node involvement, adrenal involvement, follow-up period, presence of metastases and immunohistochemical EPO expression and its characteristics.

\section{TMA Construction}

Paraffin blocks and their respective slides were selected to create the TMA. Two cylinders $(1 \mathrm{~mm}$ in diameter) of the most representative areas of the tumour were obtained from each donor block and transferred to construct the TMA. Tissue known to be positive or negative for EPO expression was used as an internal control. When present and viable, areas of non-tumourous renal tissue at least $1.0 \mathrm{~cm}$ from the primary tumour were also selected. Sequential $4-\mu \mathrm{m}$ slices of the TMA were obtained and stained with haematoxylin and eosin to confirm the diagnosis. These sections were then used in the immunohistochemical EPO assay.

\section{Immunohistochemistry}

The immunohistochemical reactions were performed in an automated Ventana Benchmark XT (Roche Diagnostics, Mannheim, Germany) after standardisation of the technique. The slide 
deparaffinisation was performed by applying EZPrep (Roche) and heating to $75^{\circ} \mathrm{C}$ for 8 minutes. Cell Conditioner (Roche) was applied for 8 minutes at $95^{\circ} \mathrm{C}$ and then for 64 minutes at $100^{\circ} \mathrm{C}$ for antigen retrieval. Endogenous peroxidase activity was blocked by applying UV DAB $\mathrm{H}_{2} \mathrm{O}_{2}$ (hydrogen peroxide 10 vol.). The slides were then incubated with a primary rabbit polyclonal anti-EPO antibody (Abcam, Cambridge, MA, USA), 1:200, for 32 minutes. As a control, 31 kidney tissue samples of non-tumourous tissue that had been included in the TMA were used. The viewing system used to immunohistochemically detect the evaluated marker was the iVIEWTM DAB Detection Kit (Ventana Medical Systems Inc., Tucson, AZ, USA).

The reactions were analysed by an experienced pathologist (LS) blinded to the disease evolution of the patients. It should be noted that because these antibodies have not been widely studied and there is a wide range of standardisation in the literature, the reaction quality was rigorously analysed in two slides from each patient, and doubtful cases were excluded. Given the pattern of EPO expression and to avoid the subjectivity inherent in the method, the reactions were evaluated in a qualitative manner. To achieve the best interpretation, the categories of "negative expression" and "positive expression" were used to define the absence or presence, respectively, of EPO expression according to immunohistochemical analysis.

\section{Statistical analysis}

SPSS for Windows, version 21.0 (SPSS Inc., Chicago, IL, USA) was used for statistical analyses. The clinical and pathological variables and the immunohistochemical expression of EPO were compared using the Pearson chi-squared test. Variables with an expected frequency of less than 5 were analysed using Fisher's exact test. The Mann-Whitney and Kruskal-Wallis tests were used to compare means among different EPO expression groups.

Overall survival (OS) was defined as the time interval between surgery and the date of death or the last visit. Disease-specific survival (DSS) was defined as the interval between primary surgery and death from RCC or the last follow-up visit. The probability of OS and DSS within 5 years was calculated using the Kaplan-Meier method, and categories were compared using the log-rank test. The Cox proportional hazards model was used to determine which variables influenced survival. The variables that significantly impacted survival in univariate analyses were included in multivariate analyses. The confidence interval was $5 \%$.

\section{RESULTS}

Of the 176 patients considered, 61 (34.6\%) patients underwent partial nephrectomy, and 115 (65.3\%) patients were treated with radical nephrectomy. The median age was 57 years (range 29 to 86 years). The median follow-up period was 48 months. Approximately half of the patients (86) had incidental tumours, and 21 (11.9\%) were diagnosed with metastasis. The mean tumour size was $6.86 \mathrm{~cm}$ (largest diameter).

EPO exhibited a predominantly cytoplasmic and homogeneous pattern of immunohistochemical expression (Fig. 1). Of all the evaluated tumours, 47 cases $(26.7 \%)$ were negative for EPO expression, and $129(73.3 \%)$ cases were positive. A total of 31 benign renal tissue samples adjacent to the tumour were also analysed. The proportion of cases positive

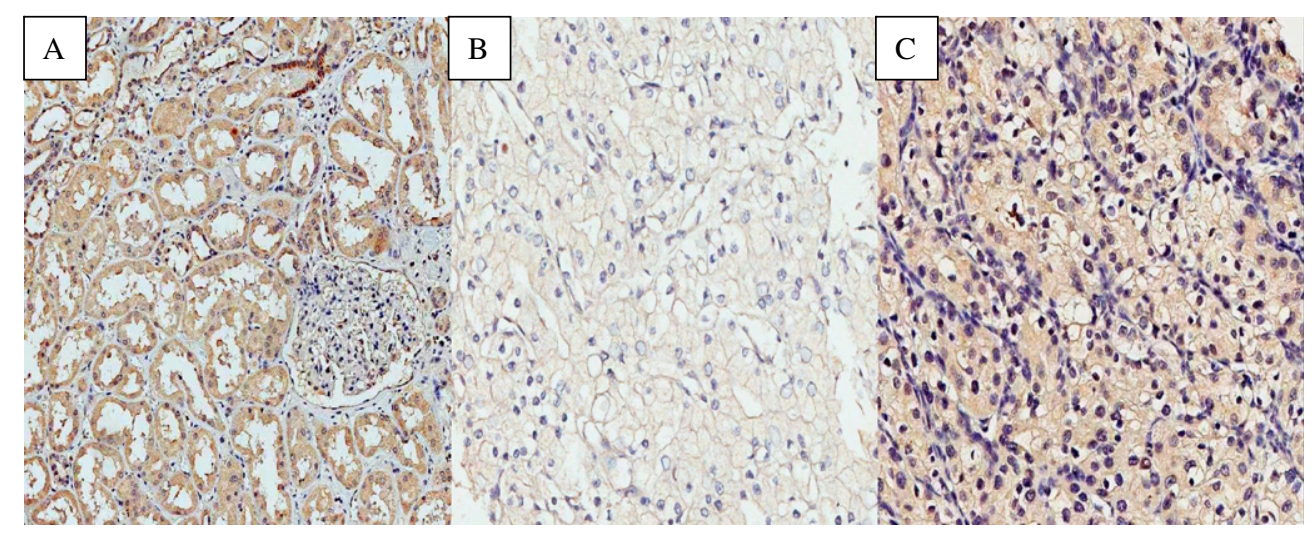

Fig. 1. Photomicrographs of immunohistochemical expression of EPO. (A) Positive expression of EPO in non-neoplastic renal cortex. (B) Negative expression of EPO in ccRCC. (C) Positive expression of EPO in ccRCC. Original magnification: $400 \mathrm{X}$. 
Table 1

Patients and pathological characteristics and association with EPO expression

\begin{tabular}{|c|c|c|c|}
\hline Variable & $\begin{array}{c}\text { Negative } \\
\text { expression } \\
n(\%)\end{array}$ & $\begin{array}{c}\text { Positive } \\
\text { expression } \\
n(\%)\end{array}$ & $P$ value \\
\hline Median age & 66.67 & 56.29 & 0.003 \\
\hline \multicolumn{4}{|l|}{ Gender } \\
\hline Male & $27(57.4)$ & $80(62)$ & \\
\hline Female & $20(42.6)$ & $49(38)$ & 0.352 \\
\hline \multicolumn{4}{|l|}{ KPS } \\
\hline$<80$ & $22(46.8)$ & $17(13,2)$ & \\
\hline$\geq 80$ & $25(53.2)$ & $112(86.8)$ & 0.016 \\
\hline \multicolumn{4}{|l|}{ Incidental tumors } \\
\hline No & $16(34)$ & $70(54.2)$ & \\
\hline Yes & $30(64)$ & $59(45.8)$ & 0.016 \\
\hline \multicolumn{4}{|l|}{ Tumor size } \\
\hline$<7 \mathrm{~cm}$ & $22(46.8)$ & $87(67.4)$ & \\
\hline$\geq 7 \mathrm{~cm}$ & $25(53.2)$ & $42(32.6)$ & 0.015 \\
\hline \multicolumn{4}{|l|}{ Transfusion } \\
\hline No & $27(57.4)$ & $105(81.4)$ & \\
\hline Yes & $17(42,6)$ & $24(18.6)$ & 0.009 \\
\hline \multicolumn{4}{|c|}{ Preoperative hemoglobin } \\
\hline$<12$ & $21(44.7)$ & $34(26.4)$ & \\
\hline$\geq 12$ & $26(55.3)$ & $95(73.6)$ & $\mathbf{0 . 0 2 7}$ \\
\hline \multicolumn{4}{|l|}{ Clinical stage } \\
\hline I or II & $27(55,1)$ & $77(60,6)$ & \\
\hline III or IV & $22(44,9)$ & $50(39,4)$ & 0.514 \\
\hline \multicolumn{4}{|l|}{ pN status } \\
\hline NO or Nx & $43(91.5)$ & $120(93)$ & \\
\hline N1 & $4(8.5)$ & $9(7)$ & 0.495 \\
\hline \multicolumn{4}{|l|}{ Metastasis } \\
\hline No & $40(85.1)$ & $114(89.1)$ & \\
\hline Yes & $7(14.9)$ & $14(10.9)$ & 0.446 \\
\hline \multicolumn{4}{|c|}{ Fat/Sinus invasion } \\
\hline No & $31(66)$ & $97(75.2)$ & \\
\hline Yes & $16(34)$ & $32(24.8)$ & 0.253 \\
\hline \multicolumn{4}{|l|}{ Fuhrman grade } \\
\hline Low (I/II) & $10(21.3)$ & $29(22.5)$ & \\
\hline High (III/IV) & $37(78.7)$ & $100(77.5)$ & 0.521 \\
\hline \multicolumn{4}{|l|}{ MVI } \\
\hline No & $7(87.1)$ & $85(80.2)$ & \\
\hline Yes & $1(12.5)$ & $21(19.8)$ & 0.613 \\
\hline \multicolumn{4}{|l|}{ Necrosis } \\
\hline No & $24(52.2)$ & $75(58.1)$ & \\
\hline Yes & $22(47.8)$ & $54(41.9)$ & 0.483 \\
\hline \multicolumn{4}{|l|}{ Adrenal } \\
\hline No & $47(100)$ & $118(91.5)$ & \\
\hline Yes & $0(0)$ & $11(8.5)$ & 0.038 \\
\hline
\end{tabular}

MVI; microvascular invasion.

for EPO expression was higher in the benign renal tissue samples than in the tumours, at $96.8 \%$ and $73.7 \%$, respectively $(p=0.002)$

The associations of the immunohistochemical expression of EPO and the main clinical and pathological variables are listed in Table 1. The median ages of the patients who were positive and negative for EPO expression were 56.2 years and 66.6 years, respectively $(p=0.003)$. Associations were also observed for EPO expression and incidental tumour presence $(p=0.016)$, Karnofsky Performance Score (KPS) $>80 \quad(p=0.016)$, tumour size $<7.0 \mathrm{~cm} \quad(p=0.015)$, the need for a blood transfusion $(p=0.009)$, higher pre-operative haemoglobin values $(p=0.027)$, and adrenal involvement $(p=0.038)$.

At the end of the follow-up period, 40 patients had died, and $75 \%$ of these deaths were due to the cancer. The OS and DSS rates of the entire sample after 5 years were $77.4 \%$ and $83.1 \%$, respectively. A univariate analysis indicated that clinical and pathological variables such as symptoms at diagnosis, KPS, ECOG status, ASA classification, clinical stage, tumour size, metastasis at diagnosis, lymph node invasion, necrosis, and fat/sinus invasion affected survival rates. Analysis of the studied marker revealed that EPO expression positively affected $\mathrm{OS}(p<0.001)$ and DSS $(p=0.044)$. The OS rates at 5 years in patients who were positive and negative for EPO expression were $82.9 \%$ and $60.9 \%$, respectively. The 5-year DSS rates of patients who were positive and negative for EPO expression were $85.3 \%$ and $76.1 \%$, respectively (Fig. 2).

In the multivariate analysis, the variables remaining as independent predictors of OS were the presence of metastasis at diagnosis $(p<0.001)$, ASA classification $(p=0.022)$ and clinical stage $(p=0.026)$. The predictive factors for the risk of cancer death (DSS) were the presence of metastasis at diagnosis $(p<0.001)$ and clinical stage $(p=0.006)$. In addition to these factors, negativity for EPO expression remained an independent predictor of the risk of death, and negatively affected OS (HR 9.67; 95\% CI $3.45-27.10 ; p<0.001)$ and DSS (HR 9.54; 95\% CI 3.04-29.92; $p<0.001$ ) (Table 2).

\section{DISCUSSION}

In the tumour microenvironment, tissue hypoxia acts as a regulatory factor inhibiting or stimulating the expression of specific genes, especially those relating to angiogenesis and cell proliferation $[17,18]$. EPO production is mediated via the intracytoplasmic accumulation of hypoxia-inducible factor $1 \alpha$ (HIF-1 $\alpha$ ), characterising the von Hippel-Lindau (VHL)-HIF-EPO pathway as the main adaptive mechanism in response to hypoxic conditions. In addition to hypoxia, another mechanism that leads to the stabilisation of HIF- $1 \alpha$ in the cytoplasm, with the subsequent increased production of these angiogenic factors, occurs via VHL gene deletions and 

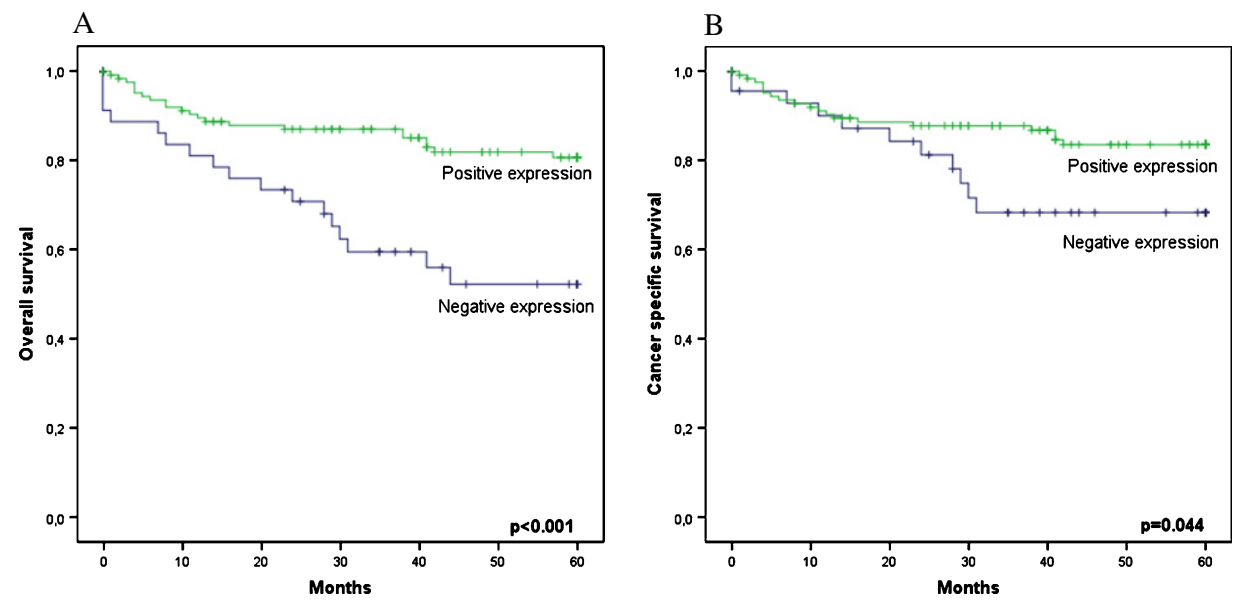

Fig. 2. Survival analysis based on EPO epression. (A) 5-years OS curves (B) 5-year CSS curves.

Table 2

Cox regression analysis for overall survival (OS) and disease-especific survival (DSS)

\begin{tabular}{|c|c|c|c|c|c|c|c|c|}
\hline \multirow[t]{3}{*}{ Feature } & \multicolumn{4}{|c|}{ 5-year OS } & \multicolumn{4}{|c|}{ 5-year DSS } \\
\hline & \multicolumn{2}{|c|}{ Univariate } & \multicolumn{2}{|l|}{ Multivariate } & \multicolumn{2}{|c|}{ Univariate } & \multicolumn{2}{|l|}{ Multivariate } \\
\hline & HR & $P$ value & HR $(95 \%$ CI $)$ & $P$ value & HR & $P$ value & HR $(95 \%$ CI $)$ & $P$ value \\
\hline \multicolumn{9}{|l|}{ EPO expression } \\
\hline Positive & \multicolumn{2}{|c|}{1.0 (Reference) } & \multicolumn{2}{|l|}{1.0 (Reference) } & \multicolumn{2}{|c|}{1.0 (Reference) } & \multicolumn{2}{|l|}{1.0 (Reference) } \\
\hline Negative & 3.026 & $<0.001$ & $9.673(3.452-27.104)$ & $<0.001$ & 2.111 & 0.044 & $9.543(3.044-29.923)$ & $<0.001$ \\
\hline MVI & 1.891 & 0.184 & $0.849(0.191-3.765)$ & 0.829 & 2.284 & 0.095 & $1.318(0.692-10.752)$ & 0.796 \\
\hline Necrosis & 2.281 & 0.011 & $0.772(0.224-2.656)$ & 0.682 & 3.188 & 0.003 & $1.345(0.405-4.468)$ & 0.628 \\
\hline Metastasis & 10.348 & $<0.001$ & $9.030(3.175-25.682)$ & $<0.001$ & 14.871 & $<0.001$ & $8.280(2.922-23.465)$ & $<0.001$ \\
\hline Lymph nodes invasion & 3.051 & 0.020 & $0.963(0.124-7.506)$ & 0.972 & 4.247 & 0.003 & $1.638(0.239-11.208)$ & 0.615 \\
\hline $\begin{array}{l}\text { Symptomatic vs Incidental } \\
\text { tumors }\end{array}$ & 3.677 & 0.001 & $1.189(0.300-4.716)$ & 0.806 & 3.459 & 0.005 & $1.044(0.221-4.920)$ & 0.957 \\
\hline ASA (3-4 vs 1-2) & 2.085 & 0.027 & $3.589(1.204-10.697)$ & 0.022 & 2.219 & 0.036 & $1.283(0.228-7.412)$ & 0.780 \\
\hline ECOG PS (1+2 vs 0$)$ & 3.050 & 0.010 & $0.501(0.132-1.901)$ & 0.310 & 2.847 & 0.025 & $1.799(0.628-5.152)$ & 0.274 \\
\hline $\operatorname{KPS}(\geq 80$ vs $<80)$ & 0.174 & 0.001 & $0.512(0.139-1.892)$ & 0.316 & 0.197 & 0.004 & $0.840(0.113-6.229)$ & 0.865 \\
\hline Tumor size $(>7 \mathrm{~cm}$ vs $<7 \mathrm{~cm})$ & 3.409 & $<0.001$ & $3.979(0.772-20.508)$ & 0.099 & 6.025 & $<0.001$ & $1.415(0.323-6.205)$ & 0.645 \\
\hline Clinical stage (III/IV vs I/II) & 3.187 & $<0.001$ & $3.972(1.178-13.390)$ & 0.026 & 5.215 & $<0.001$ & $9.589(1.890-47.639)$ & 0.006 \\
\hline
\end{tabular}

$\mathrm{HR}$, hazard ratio; CI, confidence interval; MVI, microvascular invasion.

methylation. These changes in the VHL gene occur almost exclusively in ccRCC cases [19].

EPO is a glycoprotein of the class I cytokine family and is produced by peritubular endothelial cells of the renal cortex in adults. The main function of EPO is the control of erythropoiesis in the bone marrow [20]. In non-haematopoietic tissues, the interaction of EPO with its membrane receptor (EPO-R) leads to the inhibition of apoptosis, the promotion of cell proliferation, resistance to certain drugs, and the stimulation of angiogenesis [10].

The first study that evaluated the immunohistochemical expression of EPO in only 14 RCC (ccRCC and papillary RCC) tissues fixed in $10 \%$ formalin and processed in paraffin was conducted in 1998 [21]. In that study, EPO expression was detected via immunohistochemistry in $74 \%$ of the RCC samples. Another study also revealed the immunohistochemical expression of EPO and the EPO-R in RCC patients, demonstrating strong staining in more than $90 \%$ of the samples [22]. In the present study, 129 $(73.3 \%)$ cases were positive for EPO expression, and $26.7 \%$ of the cases were negative. It should be noted that samples with no substantial degree of marker expression were considered negative, which methodologically decreases the subjectivity of the analyses and immunohistochemical patterns.

Few studies have evaluated EPO expression in RCC, although with conflicting results and baseline variables [15]. Furthermore, the evaluation of the immunohistochemical expression of EPO in normal and tumourous tissue and the associations with 
major clinical and pathological variables, especially the impact on survival, has been rarely explored in the literature.

Our study evaluated EPO expression in RCC and adjacent benign tissue $(1.0 \mathrm{~cm}$ from the tumour), demonstrating a greater proportion of cases that were positive for EPO expression in non-tumourous renal tissue than in tumours, at $96.8 \%$ and $73.7 \%$, respectively. Recently, other authors have also evaluated the immunohistochemical expression of EPO in a variety of malignant and benign tumour cell lines, using TMAs [23]. In contrast to the present study, there was no statistically significant difference in EPO expression between benign renal tissue and RCCs. It should be noted that in that study, the benign renal tissue was not adjacent to the primary tumour and was therefore not subject to the influence of the tumour microenvironment. Hypothetically, in our series, the effect of tumour cytokines on adjacent benign tissue could have activated mechanisms adaptive to hypoxia, leading to EPO expression in tissues with a normal phenotype compared to that of RCC.

Associations of the main prognostic factors of RCC and EPO expression were also evaluated in our study. The proportion of patients who were positive for EPO expression was greater in patients with incidental tumours, in those with a KPS $\geq 80$, in those whose tumours were $<7.0 \mathrm{~cm}$, in those with a preoperative haemoglobin level $\geq 12 \mathrm{~g} / \mathrm{dl}$ and in those not transfused at the time of nephrectomy. Furthermore, patients who were positive for EPO expression were younger than those who did not express EPO.

In a recent study, a number of prognostic factors in RCC patients of various histological types were evaluated. In contrast to our study, no associations of EPO expression with the main clinical and pathological variables were observed. Another study involving 249 patients with ccRCC found that EPO expression was associated with high-grade tumours [24]. In the present study, the only pathological factor that was associated with EPO expression was adrenal invasion.

The association between the immunohistochemical expression of EPO and the impact on survival is still uncertain. In the literature, one single study was able to demonstrate in a univariate analysis the impact of EPO expression on the survival of 113 patients with ccRCC. In that study, Michael et al. [25] reported that the risk of death in patients positive for EPO expression was approximately twice as high as that for patients who were negative for expression (HR $2.34 ; 95 \%$ CI $1.27-4.3 ; p=0.006)$. However, in a multivariate analysis, only the presence of macroscopic capsular invasion remained as an independent factor in survival.

In our series, patients who were positive for EPO expression had a better prognosis, and $82.9 \%$ were alive after 5 years. In contrast to the findings by Michael et al. [25], patients negative for expression were twice as likely to die from RCC (HR 2.11; 95\% CI $1.00-4.44 ; p=0.049)$. Moreover, the multivariate analysis indicated that negativity for EPO expression remained an independent predictor of worse DSS and OS rates.

It is important to note that the tumours of patients who were in better general condition (younger, with incidental tumours and better performance status and without anaemia) expressed EPO in greater proportions, which may indirectly have contributed to the better survival rates in the present study. Thus, EPO might be a more sensitive marker for patientdependent factors than for factors directly related to the disease.

Disagreements with the literature can be explained as follows: these markers are rarely studied in the literature; there are different methods of reading the results; and difficulties, such as a loss of antigenicity of the material and inadequate fixation in formalin, are inherent in the immunohistochemistry method itself. Furthermore, it is known that ccRCC has heterogeneous characteristics [26], and the immunohistochemical expression pattern of EPO may not be homogeneous throughout the tumour. To reduce the influence of intratumour heterogeneity, we evaluated two slides (containing two tumour samples) from each patient. Each slide was sectioned at different height levels of the tumour block, resulting in quadruplicate samples per patient.

In addition, EPO is also produced by the contralateral kidneys and the liver (in small amounts), and its production and serum levels may be affected by several conditions, such as general health status, comorbidities, nutritional status, dietary habits and iron levels. Regardless of these potential external or systemic influences, our findings show that intratumour EPO immunostaining is an independent prognostic factor for survival. In the future, we could design studies testing the prognostic value of this marker in samples from percutaneous small renal mass biopsies. We imagine that EPO could also be tested as a predictive factor for drug responses in ccRCC patients with metastasis.

In conclusion, our study demonstrated in an unprecedented way that the absence of EPO tissue 
expression negatively affected survival rates and remained an independent predictor of OS and DSS. Future studies may jointly evaluate immunohistochemical, serum and biomolecular expression levels to validate our findings and confirm the role of EPO as a possible marker for ccRCC patients.

\section{ACKNOWLEDGMENTS}

Urology Division, Department of Pelvic Surgery, AC Camargo Hospital.

Department of Urology, Pasteur Hospital.

\section{CONFLICTS OF INTEREST}

The authors have no conflict to report.

\section{REFERENCES}

[1] Chow WH, Devesa SS, Warren JL, Fraumeni JF Jr. Rising incidence of renal cell cancer in the United States. JAMA 1999;281:1628-31.

[2] Vaishampayan UN, Do H, Hussain M, Schwartz K. Racial disparity in incidence patterns and outcome of kidney cancer. Urol 2003;62:1012-7.

[3] Hock LM, Lynch J, Balaji KC. Increasing incidence of all stages of kidney cancer in the last 2 decades in the United States: An analysis of surveillance, epidemiology and end results program data. J Urol 2002;167:57-60.

[4] Wallen EM, Pruthi RS, Joyce GF, Wise M. Kidney cancer. J Urol 2007;177:2006-18; discussion 2018.

[5] Ferlay J, Steliarova-Foucher E, Lortet-Tieulent J, Rosso S, Coebergh JW, Comber H, et al. Cancer incidence and mortality patterns in Europe: Estimates for 40 countries in 2012. Eur J Cancer. 2013;49:1374-403.

[6] Jonasch E, Futreal PA, Davis IJ, et al. State of the science: An update on renal cell carcinoma. Mol Cancer Res 2012;10:859-80.

[7] Klatte T, Rao PN, de Martino M, et al. Cytogenetic profile predicts prognosis of patients with clear cell renal cell carcinoma. J Clin Oncol 2009;27:746-53.

[8] Farrell F, Lee A. The erythropoietin receptor and its expression in tumor cells and other tissues. Oncologist 2004;9(Suppl 5):18-30.

[9] Jelkmann W, Wagner K. Beneficial and ominous aspects of the pleiotropic action of erythropoietin. Ann Hematol 2004;83:673-86.

[10] Ribatti D, Presta M, Vacca A, et al. Human erythropoietin induces a pro-angiogenic phenotype in cultured endothelial cells and stimulates neovascularisation in vivo. Blood 1999;93:2627-36.

[11] Westenfelder C, Baranowski RL. Erythropoietin stimulates proliferation of human renal carcinoma cells. Kidney Int 2000;58:647-57.

[12] Lacombe C, Da Silva JL, Bruneval P, et al. Peritubular cells are the site of erythropoietin synthesis in the murine hypoxic kidney. J Clin Invest 1988;81:620-3.

[13] Eckardt KU. Erythropoietin production in liver and kidneys. Curr Opin Nephrol Hypertens 1996;5:28-34.

[14] Westenfelder C, Biddle DL, Baranowski RL. Human, rat, and mouse kidney cells express functional erythropoietin receptors. Kidney Int 1999;55:808-20.

[15] Morais C, Johnson DW, Vesey DA, Gobe GC. Functional significance of erythropoietin in renal cell carcinoma. BMC Cancer 2013;13:14.

[16] Zequi SC, Clavijo DA. The creation, development and diffusion of the LARCG- Latin American renal cancer group. Int Braz J Urol. 2017;43:3-6.

[17] Ohh M. Ubiquitin pathway in VHL cancer syndrome. Neoplasia 2006;8:623-9.

[18] Höckel M, Vaupel P. Tumor hypoxia: Definitions and current clinical, biologic, and molecular aspects. J Natl Cancer Inst 2001;93:266-76.

[19] Kim WY, Kaelin WG, Kim WY, Kaelin WG. Role of VHL gene mutation in human cancer. J Clin Oncol 2004;15:49915004.

[20] Wen D, Boissel JP, Showers M, Ruch BC, Bunn HF. Erythropoietin structure-function relationships. Identification of functionally important domains. J Biol Chem 1994;269:22839-46.

[21] Clark D, Kersting R, Rojiani AM. Erythropoietin immunolocalization in renal cell carcinoma. Mod Pathol 1998;11:24-8.

[22] Gong K, Zhang N, Zhang Z, Na Y. Coexpression of erythopoietin and erythopoietin receptor in sporadic clear cell renal cell carcinoma. Cancer Biol Ther 2006;5:582-5.

[23] Miyake M, Goodison S, Lawton A, Zhang G, GomesGiacoia E, Rosser CJ. Erythropoietin is a JAK2 and ERK1/2 effector that can promote renal tumor cell proliferation under hypoxic conditions. J Hematol Oncol 2013;6:65.

[24] Feng CC, Ding GX, Song NH, Li X, Wu Z, Jiang HW, Ding Q. Paraneoplastic hormones: Parathyroid hormone-related protein (PTHrP) and erythropoietin (EPO) are related to vascular endothelial growth factor (VEGF) expression in clear cell renal cell carcinoma. Tumour Biol 2013;34(6):3471-6.

[25] Michael A, Politi E, Havranek E, et al. Prognostic significance of erythropoietin expression in human renal cell carcinoma. BJU Int 2007;100:291-4.

[26] Gerlinger M, Rowan AJ, Horswell S, et al. Intratumor heterogeneity and branched evolution revealed by multiregion sequencing. N Engl J Med 2012;366:883-92. 